\title{
An Efficient Biogeography based Face Recognition Algorithm
}

\author{
Daya Gupta \\ Delhi Technological University \\ Computer Engineering Department \\ Delhi, India \\ e-mail:dgupta@dce.ac.in
}

\author{
Lavika Goel \\ Delhi Technological University \\ Computer Engineering Department \\ Delhi, India \\ e-mail: goel_lavika@yahoo.co.in
}

\author{
Abhishek \\ Delhi Technological University \\ Computer Engineering Department \\ Delhi, India \\ e-mail: abhi_umb@yahoo.com
}

\begin{abstract}
Extracting the optimal features from images is always required in face recognition algorithm to achieve high accuracy. In this paper we have presented an efficient face recognition algorithm based on Biogeography Based Optimization (BBO). First we extract the features using the principal Component Analysis (PCA) after applying gabor filters and then we apply $B B O$ to get the most desirable features. The proposed biogeography based face recognition algorithm is applied to search the most desirable features based on well defined fitness function. Performance analysis is performed using Olivetti Research Laboratory (ORL) face database. Performance results show that biogeography based face recognition algorithm generates better results than the original PCA technique with gabor filters.
\end{abstract}

Keywords-Face Recognition, Biogeography Based Optimization, PCA, Gabor Filter

\section{INTRODUCTION}

There is a lot of research avenues in the field of face recognition due to challenges present in the field. The goal of face recognition is to match a given image against a large database of images to check its presence. The face recognition has been applied to two most important applications i.e verification (one to one matching) and identification (one to many matching) [9]. Researchers have presented a lot of techniques for face recognition [1]. These techniques can be categorized as holistic matching method for e.g Principal Component Analysis (PCA) and local feature matching method [2]. In this paper we will focus on PCA. One of the biggest challenges in PCA is that we cannot use it on raw images directly. They need to be properly aligned and uniformly illuminated. This challenge can be solved using gabor filters which takes a raw image, generates gabor filter response and convert the raw image into properly aligned and constantly illuminated image. We have explored the research that showed that Gabor PCA based method for face recognition outperforms PCA based Eigenface method [3]. To improve further this hybrid technique we applied BBO to further optimize the desirable features from the image and go on to illustrate that in our results.

\section{A. Principal Component Analysis}

Extraction of features from the images is the foremost step in face recognition. PCA is a standard technique used for feature extraction. The image in the form of $\mathrm{A} \times \mathrm{A}$ matrix can be expressed as a point in the space $\mathrm{A} \times \mathrm{A}$ dimensions. The goal of PCA is to find the required vectors that can represent the image information and form another space. PCA steps are as follows:

1. Let the training set of face images be $\Gamma_{1}, \Gamma_{2}, \Gamma_{3}, \ldots \ldots \ldots, \Gamma_{p}$.

2. We calculate the average face of the set by $\quad \psi=$ $\frac{1}{p} \sum_{r=1}^{p} \Gamma_{r}$

3. We calculate the difference of each face from the average by vectors $\phi=\Gamma_{i}-\psi$.

4. We calculate eigenvalues and eigen vectors from covariance matrix as shown (1) and a new face image is transformed into its face components by this operation [2].

$$
c=\frac{1}{p}\left[\sum_{R=1}^{p} \emptyset_{R} \emptyset_{R}^{T}\right]
$$

5. We calculate weights using (2) and from them we form a vector $\Omega=\omega_{1}, \omega_{2}, \ldots \omega_{p}$ that represents the contribution of each eigenvector in representation of input face image.

$$
\omega_{k}=\mu_{K}^{T}(\Gamma-\psi), \mathrm{k}=1,2, \ldots . \mathrm{P}
$$

where $\mu_{k}$ is eigen vector.

These weights may be used in a face classification algorithm to find which of predefined face classes that describe the face [12].

\section{B. Gabor Filters}

Gabor filters generally called as Gabor wavelets or kernels are complex band pass filters. They have shape similar to the shape of the cells of the visual cortex of mammalian brains. These are used in many applications such as extraction of multiresolutional, spatially local features of a confined frequency band [5]. Another important feature of the $=$ Gabor filters is that they act as an efficient tool for facial feature extraction and robust face recognition [3]. In general the family of 2D Gabor filters can be defined in the spatial domain in the following manner. $[4,6,7,8,9]$ :

$$
\begin{gathered}
\psi_{u, v}(a, b)=\frac{f_{u}^{2}}{\pi \mathrm{K} \eta} \mathrm{e}^{-\left(\left(f_{u}^{2} / k^{2}\right) a^{2}+\left(f_{u}^{2} / \eta^{2}\right) b^{2}\right)} \mathrm{e}^{j 2 \pi f_{u} a^{\prime}} \\
\text { Where } a^{\prime}=a \cos \theta_{v}+b \sin \theta_{v}, \quad b^{\prime}=-a \sin \theta_{v}+ \\
b \cos \theta_{v}, f_{u}=f_{\max } / 2^{(u / 2)} \text { and } \theta_{v}=v \pi / 8 .
\end{gathered}
$$

Each gabor filter represents a gaussian kernel function modulated by complex plane wave whose center frequency and orientation are given by $f u$ and $\theta v$, respectively. The parameter $\kappa$ and $\eta$ determine the ratio between center frequency and size of Gaussian envelope. Though we can have different values for above mentioned parameters determining characteristics of the filters, the most common 
parameters used for face recognition are $\kappa=\eta=\sqrt{ } 2$ and $f \max =0.25[6,7,8,9]$. When using gabor filters for facial feature extraction, researchers typically construct a filter bank featuring filter of five scale and eight orientation (comprised of 40 filters as shown in Fig. 1), that is $\mathrm{u}=$ $0,1 \ldots \ldots \mathrm{p}-1$ and $\mathrm{v}=0,1 \ldots, \mathrm{r}-1$, where $\mathrm{p}=5$ and $\mathrm{r}=8[4]$.

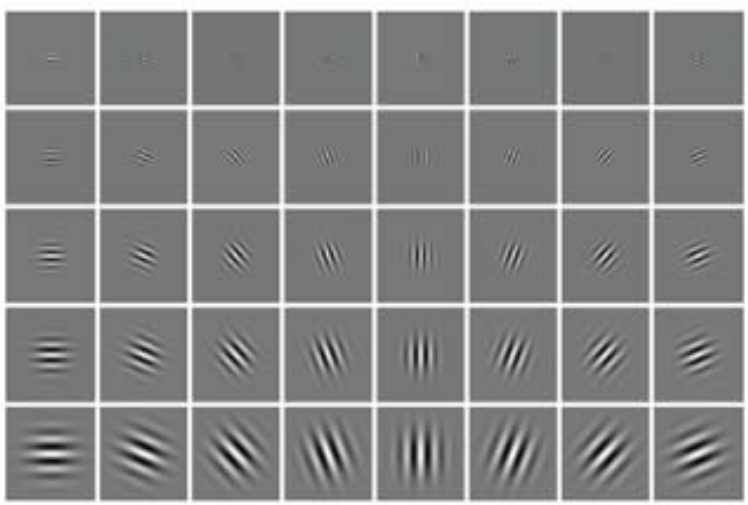

Figure 1. The real parts of the Gabor filter bank commonly used for feature extraction in the field of face recognition [4].

\section{Pricipal Component Analysis of gabor filters}

First we need to apply gabor filter to the raw images to generate gabor filter response and then these gabor filter response act as input to the PCA. The whole process till now is shown in the Fig. 2. Gabor filter provides robustness against varying contrast and brightness. It can also represent characteristic of local face area.

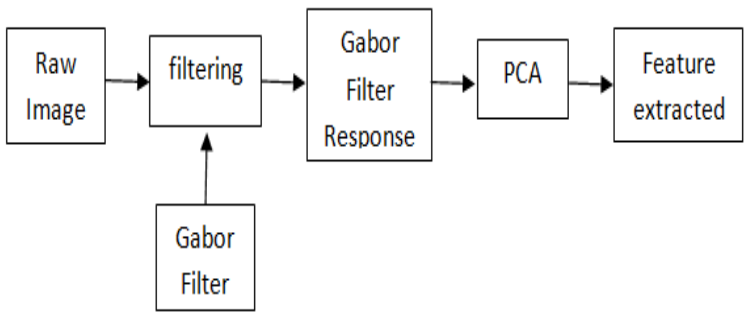

Figure2. Features extraction

Let $\mathrm{D}(\mathrm{a}, \mathrm{b})$ be a grey scale image of size $\mathrm{m} \times \mathrm{n}$ pixels and let $\psi_{u, v}(a, b)$ denote a gabor filter given by its center frequency fu and orientation $\theta \mathrm{v}$. The filtering operation of the given face image $\mathrm{D}(\mathrm{a}, \mathrm{b})$ can be defined as $[4,6,7,8,9]$

$$
M_{u, v}(a, b)=D(a, b) * \Psi_{u, v}(a, b)
$$

Where $M_{u, v}(a, b)$ denote the gabor filter response which can be decomposed into real $\left(E_{u, v}(a, b)\right)$ and imaginary part $\left(O_{u, v}(a, b)\right)$.

$$
\begin{aligned}
& E_{u, v}(a, b)=\operatorname{R\varepsilon }\left[M_{u, v}(a, b)\right] \\
& O_{u, v}(a, b)=\operatorname{Im}\left[M_{u, v}(a, b)\right]
\end{aligned}
$$

\section{Selecting Optimal features using $B B O$}

We extract the features using the principal component analysis of gabor filter. But further we need to find the most optimal set of features without compromising accuracy.In the proposed algorithm we are using an evolutionary algorithm based on swarm intelligence known as BBO [14]. BBO is explained in the next section [10].

\section{E. Biogeography based optimization}

Biogeography is the study of geographical distribution of biological organisms. It aims to learn how species migrate from one island to another. Biogeography is expressed in terms of various factors such as habitat area, immigration rate, emigration rate, habitat suitability index (HSI) and suitability index variables (SIV). Each individual is considered as a "habitat" with a habitat suitability index to measure individual. Suitability index variables (SIV) are those variables of an individual by which we can characterize habitability. A good solution always has a high HSI and a poor solution has low HSI. A good solution shares its features with the poor ones using migration [11, $13,15,16]$.

\section{PROPOSED APPROACH FOR USING BBO}

After features are extracted using gabor filter and PCA. The extracted features are further minimized using $\mathrm{BBO}$. The final feature subset consists of the most optimal features. Table 1 shows some information about the BBO.

\section{A. Suitability Index Variable}

In the proposed approach the habitat represents a possible solution (optimal features). The habitat comprises of several Suitability Index Variable (SIVs). Features extracted from every single image represent a SIV.

\section{B. Habitat Suitability Index and Fitness Function}

Each habitat is evaluated on the basis of habitat suitability index (HSI). Habitat Suitability Index can be considered as a value return by the fitness function. A habitat is considered rich in features if its HSI value is closer to the ideal habitat's HSI value and if its value is far from the ideal HSI value then the habitat is considered as a poor habitat containing less features. Based on the HSI value we can always distinguish between good solution and the bad ones. In order to improve the bad solutions we can migrate certain set of selected features from the ideal solution to bad ones. We have used standard mean as the fitness function for the approach. After developing the training model we use Euclidean distance to calculate the matching score between the features of test image and that of training model.

\section{Distance Calculation}

After the development of train model from the training set images, we calculate the similarity matrix using the matrix which contains the features extracted from test set of images. The distance used to compute the similarity matrix is 'Euclidean'. Euclidean distance is the straight line 
distance between two points present in a N-dimensional space. For example, the Euclidean distance between any two points $x_{i}$ and $y_{i}$ present in N-dimensional space is given by [10]:

$$
D=\sqrt{\sum_{i=1}^{N}\left(x_{i}-y_{i}\right)^{2}}
$$

TABLE 1. BBO PARAMETERS

\begin{tabular}{|l|l|}
\hline Number of iterations of BBO algorithm & 120 \\
\hline SIV value & Real \\
\hline Number of SIVs in a Habitat & 120 \\
\hline
\end{tabular}

Extract the features from the training set and test set in matrices as shown in Fig. 2 using Gabor filter and PCA.Lets say 'train_data' is a matrix contains feature extracted from training set and 'test_data' contains features extracted from test set.

1. Calculate the hsi value for the whole train data using fitness function.It will be treated as ideal HSI.

2. Apply BBO for 120 (number of SIVs) iterations and in each iteration do the following :

a. Calculate HSI value for the SIV (or column) of the image present in test_data matrix.

b. Compare the calcultaed HSI value with ideal HSI.

c. If calculated HSI value is close to ideal HSI do nothing. Go to next iteration.

d. If calculated HSI is not close to ideal HSI then perform migration to improve test_data.

3. Calculate similarity matrix from train_data and test_data using euclidean distance and plot the required curve.

Figure 3: Proposed BBO Algorithm for face recognition.

\section{DATABASE AND EXPERIMENTAL SETUPS}

For our biogeography based face recognition algorithm we use standard ORL database acquired at the Olivetti Research Laboratory in Cambridge, U.K. The database consists of 400 distinct images that correspond to 40 distinct subjects. Therefore, each subject has 10 facial images each image has got different illumination, pose and facial expression. The size of each image is $92 \times 112$ pixels and has 8-bit grey levels [9]. Some of the images from the ORL database are shown below in Fig. 4.

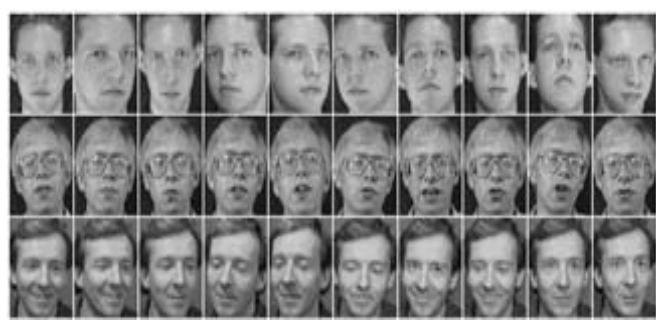

Figure 4: Representative set of ORL face database

For our experiment we have used 360 images that is six images per subject. We have divided the images of every subject into two sets. First set (training set) contains the first three images of every subject. Second set (test set) contains the next three images of every subject. So, both training set and test set consists of 120 images each. Training set trains the system and build the train model. Test set is used for assessment and calculation of certain parameters. We develop the train model from the training set by applying gabor filter and PCA and use the same techniques to extract features from test set. Both training set and features extracted from test set images are matrices of size $119 \times 120$. Each column represents an image or a SIV of length equal to 119 . Now, we apply BBO to the features extracted from the test set and use train model as ideal habitat.

\section{PERFORMANCE ANALYSIS}

For performance analysis of our biogeography based face recognition algorithm we calculate the similarity matrices, in which each of the image vector is compared with each image vector present in the train model and client and imposter matching scores are calculated. A client is an entity who is making a genuine identity claim whereas imposter is the one making a false identity claim. We calculate performance metrics from the similarity matrix like false acceptance rate (FAR), false rejection rate (FRR), half total error rate (HTER), verification rate at $1 \%, .1 \%$ and 1 , etc [4].

$$
F R R=(a / b) \times 100 \%
$$

Where $\mathrm{a}=$ number of rejected genuine identity claims and $\mathrm{b}=$ total number of genuine claims made.

$$
F A R=(c / d) \times 100 \%
$$

Where $\mathrm{c}=$ number of accepted false identity claims and

$\mathrm{d}=$ number of false identity claims made.

And HTER is given by

$$
H T E R=0.5(F A R+F R R)
$$

And verification rate is equal to $1-F R R$.

Now, in order to show the efficiency of the proposed biogeography based algorithm we have plotted the receiver operating characteristic (ROC) curve having verification rate on the $\mathrm{Y}$ - axis and False Acceptance rate at $\mathrm{X}$-axis. We have plotted two ROC curves in Fig. 5. In Fig. 5 the lower dashed line shows the ROC curve for simple gabor filter and PCA technique without applying BBO and the upper solid line shows the ROC curve for the proposed $\mathrm{BBO}$ 
based algorithm. Clearly, the proposed biogeography based algorithm shows better verification rate

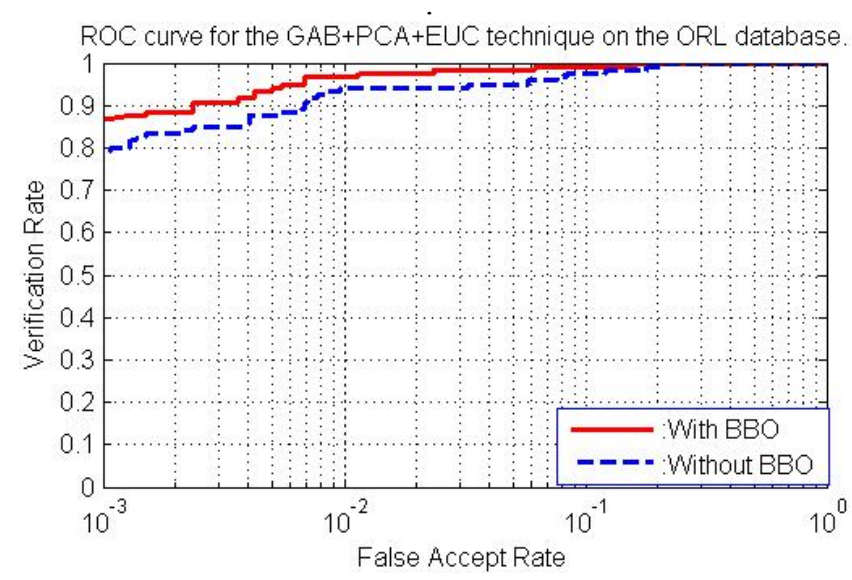

Figure 5. ROC curve for gabor and PCA technique.

\section{CONCLUSION}

In this paper, we propose an efficient biogeography based face recognition algorithm. We apply Biogeography Based Optimization (BBO) to the features extracted from test set of images after applying gabor filters and PCA as shown in Fig. 2. The proposed algorithm tries to find the most optimal set of features from the images. The performance is evaluated using the standard ORL face database. The results obtained in Fig. 5 shows that the proposed biogeography based algorithm shows better verification rate than the normal face recognition algorithm.

\section{REFERENCES}

[1] Rabia Jafri and Hamid R. Arabnia"A survey of face recognition techniques," Journal of Information Processing Systems, Vol.5, No.2, June 2009 .

[2] M. Turk and A. Pentland, "Eigenfaces for recognition. Journal of cognitive neuroscience",3:72-86,1991.

[3] D Murugan, Dr. S Arumugam, K Rajalakshmi and Manish T I "Performance Evaluation of face recognition using Gabor filter, log Gabor filter and discrete wavelet transform", International jouranal of computer science \& information Technology(IJCSIT), Vol.2,No.1,February 2010
[4] Vitomir truc and Nikola Pave ic, "The complete Gabor-Fisher classifier for robust face recognition," EURASIP journal on Advances in Signal Processing volume 2010.

[5] V. truc, B. Vesnicer, N. Pave ic, "The phase-based Gabor Fisher classifier and its application to face recognition under varying illumination conditions," in Proceedings of the $2^{\text {nd }}$ International Conference on Signal Processing and Communication Systems (ICSPCS '08), pp, 1-6, Gold Coast, Australia, December 2008.

[6] C. Liu and H. Wechsler, "Gabor feature based classification using the enhanced Fisher linear discriminant model for face recognition", IEEE Transactions on image Processing, vol. 11, no. 4, pp. 467476,2002 .

[7] L. Shen and L. Bai, "A review of Gabor wavelets for face recognition", Pattern Analysis and Applications, vol. 9, no. 2,pp.273-292,2006.

[8] L. Shen,L.Bai, and M. fairhurst, "Gabor wavelets and general discriminant analysis for face identification and verification", Image and Vision Computing,vol. 25, no. 5, pp. 553-563, 2007.

[9] Vitomir truc and Nikola Pave ic, "Gabor-based kernel partialleast squares discriminantion features for face recognition", Informatica, vol.20,no.1,pp.115-138,2009.

[10] Er. Navdeep Kaur Johal, Er. Poonam Gupta and Er. Amanpreet kaur, "Face recognition using biogeography based optimization", International Journal of Computer Science and Information Security, Vol.9,No.5,May2011.

[11] D. Simon, "Biogeography -based optimization", IEEE Transactions on Evolutionary Computation, vol. 12, no. 6, pp. 702713,2008 .

[12] Ki-Chung Chung, Seok Cheol Kee and Sang Ryong Kim, “ Face recognition using principal component analysis of Gabor filter responses".

[13] Lavika Goel, Daya Gupta and V. K Panchal, "Hybrid bio-inspired techniques for land cover feature extraction: a remote sensing perpective", Applied soft computing. Elsevier publications, pp. 832849, vol. 12(2),2011.

[14] Lavika Goel, Daya Gupta and V.K Panchal, "Information sharing in swarm intelligence techniques: a perspective application for natural terrain feature elicitation" International Journal of Computer Applications (IJCA), vol. 32,2011.

[15] Lavika Goel, Daya Gupta and V. K Panchal, " Embedding expert knowledge to Hybrid Bio-Inspired Techniques :an adaptive strategy towards focused land cover feature extraction", International Journal of Computer Science and Information Security (IJCSIS), 1(2),pp. 244-253, 2010.

[16] Lavika Goel, Daya Gupta and V. K Panchal, "Performance governing factors of BBO for land cover feature extraction : An Analytical Study, World Congress on Information and Communication Technologies (WICT) IEEE Publications, pp. 165$170,2011$. 